\title{
Back to Galilean Transformation and Newtonian Physics Refuting the Theory of Relativity
}

\section{Eisenman $\mathrm{MN}^{*}$}

Independent Researcher, Israel

\begin{abstract}
This paper refutes the theory of relativity. Previous attempts by others were based on pointing at contradictions between corollaries of the theory of relativity and reality, often called paradoxes. The main point of this article is to indicate and correct the error that led scientists at the turn of the twentieth century to formulate the faulty theory of relativity. In one of his lectures the late Professor Itzhak Bar Itzhak Z"L (Technion, Israel Institute of Technology, Haifa
\end{abstract} Israel) compared physicists and engineers by means of an equation.

Engineer=Physicist + common sense

Wherefrom it follows that:

Physicist=Engineer - common sense

As we shall demonstrate below, the theory of special relativity was born out of an error and some lack of common sense.

Many attempts have been made to refute the theory of relativity. I assume that all of them have dealt with contradictions between corollaries of the theory of relativity and reality, often called "paradoxes". The wrong rejection of two of these paradoxes, the twin paradox and the apparent instability of planetary trajectories due to gravitational acceleration delay (also termed "retardation"), based on pseudo-scientific arguments led me to realize that the only practical way to refute the theory of relativity is by displaying the error on which it is based. This error is associated with Maxwell's equations.

Maxwell's equations are a brilliant formulation of the laws of electromagnetism. However, they were derived for static systems, i.e.; where there was no motion relative to the relevant coordinate system (RCS). At the turn of the twentieth century some scientists assumed that these equations pertain also to dynamic systems, wherefrom it follows that the speed of light is constant in all inertial coordinate systems. This in turn led to the Lorentz transformation and to Einstein's theory of relativity.

This article shows that Maxwell's equations do not apply to dynamic systems where there is motion relative to the RCS. As a consequence of the correction of these equations it is proven below that the Galilean transformation and Newtonian laws of mechanics are universally valid, not just as low speed approximations.

The theory of relativity was born out of the attempt to force an incorrect form of Maxwell's equations on al electromagnetic phenomena. The formulation of the corrected Maxwell equations finally refutes the theory of relativity.

Keywords: Special relativity; Maxwell equations; Mechanics

\section{Nomenclature}
$\underline{B}:$ Magnetic Induction Vector
D: Electric Excitation Vector
$\underline{E}$ : Electric Field Strength Vector
$\boldsymbol{H}$ : Magnetic Excitation Vector

\section{$\boldsymbol{J}$ : Electric Current Density Vector}

RCS: Relevant Coordinate System: The coordinate system in which Maxwell's Equations are Expressed

$\boldsymbol{V}$ : Velocity Vector with Respect to the RCS

$\rho:$ Charge Density

$\boldsymbol{\varepsilon}$ : Dielectric Constant

$\phi$ : Magnetic Flux

$\boldsymbol{\mu}$ : Magnetic Permeability

$\boldsymbol{B}$ : Magnetic Induction Vector
$\underline{D}$ : Electric Excitation Vector

$\underline{\boldsymbol{E}}$ : Electric Field Strength Vector

$\underline{\boldsymbol{H}}$ : Magnetic Excitation Vector

$\boldsymbol{J}$ : Electric Current Density Vector

RCS: Relevant Coordinate System: The coordinate system in which Maxwell's equations are expressed

$\underline{\boldsymbol{V}}$ : Velocity Vector with Respect to the RCS

*Corresponding author: Eisenman MN, Independent Researcher, Israel, Tel: 148789455; E-mail: moshe_eisenman@yahoo.com

Received August 03, 2016; Accepted September 26, 2016; Published September 28, 2016

Citation: Eisenman MN (2016) Back to Galilean Transformation and Newtonian Physics Refuting the Theory of Relativity. J Phys Math 7: 198. doi: 10.4172/20900902.1000198

Copyright: $\odot 2016$ Eisenman MN. This is an open-access article distributed under the terms of the Creative Commons Attribution License, which permits unrestricted use, distribution, and reproduction in any medium, provided the original author and source are credited. 
$\rho:$ Charge Density

$\boldsymbol{\varepsilon}:$ Dielectric Constant

$\phi$ : Magnetic Flux

$\boldsymbol{\mu}:$ Magnetic Permeability

\section{Introduction}

As an engineer I have always had a strange feeling about the theory of special relativity. It sounds very mysterious and sophisticated, but somehow it has never sounded right. During the summer of 2011, being unemployed and approaching retirement, I started researching those issues.

While searching for the incentives that led to the theory of special relativity, i.e.: shortcomings of the classical theories of physics, I encountered the "magnet and coil" problem. Although the measured effect of a magnet on a coil depends only on the relative velocity between them - according to Maxwell's equations the effect depends also on the state of an observer, e.g.: an observer stationary with respect to the magnet sees a different effect than that viewed by an observer stationary relative to the coil. The "magnet and coil" problem is presented at the following link.

As soon as I saw it I had the feeling that there must be a problem with Maxwell's equations concerning some coordinate system transformation. And indeed, Maxwell's equations were derived for static systems where there was no motion relative to the RCS. Consequently these equations are incomplete, namely: some of their terms are missing. Their application to dynamic systems, where there is motion relative to the RCS, leads to the erroneous theory of special relativity. The assumption of the universal validity of these equations, i.e.: their applicability to dynamic systems, is false and it is very surprising that such an error has gone unnoticed for over a century.

Many people whom I ask to review the article refer me to articles which present test results that supposedly "validate" the theory of relativity. It is important to note that a validity of a theory cannot be established solely by its predictions. Even if all the predictions of a theory conform to valid test results (in the case of the theory of relativity I would certainly not bet my head on it) it may still be invalid if there is an error in the process of its derivation. Unlike Newton's second law of mechanics, which is expressed as a differential equation, Maxwell's equations are derived from more basic laws. As has been indicated in the previous paragraph - these derivations are faulty, yielding the theory of relativity invalid.

The theory of relativity is refuted by each of the following steps:

1. Two quotes from the literature are presented in chapter 1 . The first quote states that Maxwell's equations are limited to static systems (systems at rest), i.e.: where there is no motion relative to the RCS. The second quote states that Maxwell's equations are universally valid, namely: that they pertain also to dynamic systems where there is motion relative to the RCS. The discrepancy between these two quotes is the basis for the faulty theory of relativity.

2. The electromagnetic (EM) differential equations corresponding to Ampere, Faraday and charge conservation laws are derived in appendix A where it is demonstrated that Maxwell's equations are limited to static systems and that Ampere, Faraday and Gauss's laws require that the speed of propagation of electric and magnetic fields must be infinite (section A.5).
The complete set of the EM (corrected Maxwell) equations is presented in chapter 1 . It is shown that the notion of the speed of light being constant in all inertial coordinate systems stems from the wrong application of Maxwell's equations to dynamic systems. It is also pointed out that due to terms restored to the corrected Maxwell equations they do not equate under the Lorentz transformation rendering it, along with the theory of relativity which is based on this transformation, invalid.

3. A solution to the corrected Maxwell equations indicates that these equations are invariant under the Galilean transformation. Consequently the time-rate, space and mass are invariant and that velocity vectors are additive, which means that the speed of light can be exceeded.

We are thus faced with two possibilities: Either Ampere, Faraday, and Gauss's laws are valid or they are not. According to the first possibility all the corollaries of the theory of relativity are wrong. However, if some corollaries of this theory can be verified by valid experiments - then, according to the second possibility, the above mentioned laws are invalid and must be corrected. In any case, the theory of relativity is refuted.

It can be demonstrated that the "magnet and coil" problem is easily resolved by the application of the corrected Maxwell equations, as is done for the "Faraday paradox" in section A.8.

\section{Maxwell Equations, Common and Corrected Represen- tations}

How can a theory be refuted? One obvious way of refuting a theory which predicts physical phenomena is by displaying contradictions between the theory's predictions and reality. In the case of the theory of relativity there are many discrepancies between corollaries of the theory and reality. These are referred to as paradoxes. The problem is that these paradoxes have been wrongly dismissed by indoctrinated physicists on the basis of pseudo-scientific arguments. Furthermore, many and sometimes very expensive experiments have been performed to "prove" the validity of this theory, something we do not see for any other law of physics. The results of the experiments are often irrelevant because there is a logical problem with any outcome. Consequently, it seems that the only practical way to refute the theory of relativity is to point at the error on which it is based. This error is deeply rooted in the common representation of Maxwell's equations.

Before discussing the electromagnetic (EM) differential equations it is necessary to define the notions of static and dynamic systems.

In the process of formulating the EM equations reference is made to one or several coordinate systems, which are not necessarily inertial. Out of all those coordinate systems there is one with respect to which the EM equations are expressed. This coordinate system is referred to as the "relevant coordinate system" (RCS)

A static system, or a system at rest, is defined as a setup where there is no motion relative to the RCS. In other words, any surface or volume selected in the RCS and inspected at different times remains stationary, i.e.: it does not have any translational motion, it does not rotate and does not deform with respect to the RCS.

A dynamic system, or a system that is not at rest, is defined as an arrangement where at least one point can be found which moves with respect to the RCS. Since, as it turns out, the vector velocity field $\underline{V}_{\text {in }}$ the EM equations is at least once space-wise differentiable [see equations (1.1), (1.2) and (1.5)], it must be space-wise continuous. Consequently, 
a region can be found around the moving point that moves with respect to the RCS. This means that a surface $A$ and a closed volume Vol can be found which move relative to the RCS. The surface and volume may have translational motion and they may be rotating and deforming relative to the RCS.

When dealing with physical phenomena in vacuum, translation and rotation do have physical meaning. For example: Given a coordinate system that moves and rotates relative to the RCS, a surface and a volume which are stationary with respect to the moving coordinate system move and rotate relative to the RCS. Although a velocity vector field can be defined so that a surface and a volume will also deform - in vacuum the deformation does not have any physical meaning. Deformation has a meaning in relation to moving particles where the particles on a surface or inside a volume are monitored at different times. If the velocity vector field is defined as the velocity of the particles - the surface or volume which contains the particles may have translational motion, may be rotating and deforming. Examples of the most general dynamic systems are magneto-fluid dynamic systems such as the plasma flow in the fusion chamber of the Tokamak. Other examples are the astrophysical phenomena of supernovae, solar storms, etc. In these examples there exist surfaces and volumes which move, rotate and deform relative to a selected RCS.

The EM equations should be derived for the most general case of dynamic systems.

The common representation of Maxwell's equations is valid only for static systems. This is obvious from the derivation of these equations in appendix $A$. However, in order to emphasize this fact here is a quote of the third paragraph of chapter 4 (page 18) in [1]:

"We must form the time derivative of the first Eq. (1). We will here imagine the surface $\Delta \sigma$ to remain fixed, which obviously applies to media at rest, to which we shall confine ourselves initially."

This restriction, or initial confinement, to media at rest greatly facilitates the derivation of Maxwell's equations in reference 1, because it enables the replacement of the total time derivatives in Faraday's, Ampere's and charge conservation laws [equations (A.1.1), (A.2.1) and (A.3.1)] with partial time derivatives and freely interchange the order of integration and differentiation. However, this restriction limits Maxwell's equations to static systems only. The physicists at the turn of the twentieth century were unaware of this limitation. They assumed that Maxwell's equations were universally valid (i.e.: applicable to any inertial coordinate system) and tried to apply them to dynamic systems which led to inconsistencies. But instead of realizing and correcting the error (by modifying Maxwell's equations) they introduced the Lorentz transformation which was the foundation of the flawed theory of relativity. The quote of the [1] confirms the above statements:

"The path taken by Einstein in 1905 in the discovery of the special theory of relativity was steep and difficult. It led through the analysis of the concepts of time and space and some ingenious imaginary experiments. The path that we shall take is wide and effortless. It proceeds from the universal validity of the Maxwell equations and the tremendous accumulation of experimental material on which they are based. It ends almost inadvertently at the Lorentz transformation and all its relativistic consequences." [Remark: the different font in the above two quotes appears in the original text of reference [1].

The basis for the erroneous theory of relativity is the discrepancy between the two above mentioned quotes: The first quote states that Maxwell's equations are limited to static systems, while the second quote assumes that these equations are universally valid, i.e.: they apply also to dynamic systems.

In this chapter the electromagnetic laws are presented as a set of eight partial differential equations. Only three of those equations, corresponding to Faraday's, Ampere's and charge conservation laws, contain time derivatives. We proceed with the derivation of the differential equations corresponding to these three laws in appendix $\mathrm{A}$ and the presentation of the complete set of differential equations governing electromagnetic phenomena. Maxwell's equations are a reduced form of the general EM equations when the velocity vector $\underline{V}=0$ everywhere, thus apply to static systems only. It is then demonstrated that the assumption of their universal validity (i.e.: their wrong application to dynamic systems) leads to the notion of the speed of propagation of electromagnetic waves being constant in all inertial coordinate systems, hence to the Lorentz transformation and Einstein's erroneous theory of relativity.

The complete set of the corrected electromagnetic differential equations is presented in equations (1.1) to (1.8). The equations corresponding to Ampere's, Faraday's and charge conservation laws, in their most general form (i.e.: applicable to dynamic systems), are derived in appendix A. Equations (A.1.11), (A.2.7) and (A.3.8) are rewritten as equations (1.1), (1.2) and (1.5), respectively.

$$
\begin{aligned}
& \frac{\partial \underline{B}}{\partial t}+(\underline{V} \cdot \nabla) \underline{B}=-(\nabla \times \underline{E})+[(\underline{B} \cdot \nabla) \underline{V}-(\nabla \cdot \underline{V}) \underline{B}] \text { Faraday's law } \\
& \frac{\partial \underline{D}}{\partial t}+(\underline{V} \cdot \nabla) \underline{D}+\underline{J}=(\nabla \times \underline{H})+[(\underline{D} \cdot \nabla) \underline{V}-(\nabla \cdot \underline{V}) \underline{D}] \text { Ampere's law } \\
& \nabla \cdot \underline{B}=0 \text { Gauss's magnetic flux law } \\
& \nabla \cdot \underline{D}=\rho \text { Gauss's electric flux law } \\
& \frac{\partial \rho}{\partial t}+(\underline{V} \cdot \nabla) \rho+\rho(\nabla \cdot \underline{V})+\nabla \cdot \underline{J}=0 \text { Charge conservation law } \\
& \underline{J}=\sigma \underline{E} \\
& \underline{D}=\varepsilon \underline{E} \\
& \underline{B}=\mu \underline{H}
\end{aligned}
$$

The common formulation of Maxwell's equations is valid only for a stationary case, i.e.: where there is no motion relative to the RCS, namely:

$\underline{V}=0$

Equations (1.1), (1.2) and (1.5) along with (1.9) yield the common Maxwell's equations corresponding to Faraday's, Ampere's and charge conservation laws:

$$
\begin{aligned}
& \frac{\partial \underline{B}}{\partial t}=-\nabla \times \underline{E} \text { Faraday's law } \\
& \frac{\partial \underline{D}}{\partial t}+\underline{J}=\nabla \times \underline{H} \text { Ampere's law } \\
& \frac{\partial \rho}{\partial t}+\nabla \cdot \underline{J}=0 \text { Charge conservation law }
\end{aligned}
$$

We proceed to solve the common Maxwell's equations (1.10) to (1.12). Limiting ourselves to an isotropic and non-conducting medium (such as outer space or vacuum) we have:

$$
\begin{aligned}
& \rho=0 ; \quad \sigma=0 ; \quad \underline{J}=0 \\
& \nabla \cdot \underline{E}=0 ; \quad \nabla \cdot \underline{H}=0 ; \quad \varepsilon=\varepsilon_{0} ; \quad \mu=\mu_{0} ;
\end{aligned}
$$


So in this case (vacuum), substituting equations (1.8) and (1.7) into equations (1.10) and (1.11), respectively, Maxwell's equations attain the following form:

$$
\begin{aligned}
& \mu \frac{\partial \underline{H}}{\partial t}=-\nabla \times \underline{E} \\
& \varepsilon \frac{\partial \underline{E}}{\partial t}=\nabla \times \underline{H}
\end{aligned}
$$

Equations (1.15) and (1.16) are Maxwell's equations for outer space (vacuum).

From equation (1.15) and (1.16) we obtain:

$$
\frac{\partial \underline{H}}{\partial t}=-\frac{1}{\mu} \nabla \times \underline{E} ; \quad \varepsilon \frac{\partial^{2} \underline{E}}{\partial t^{2}}=\nabla \times \frac{\partial \underline{H}}{\partial t}
$$

It follows from equation (1.17):

$$
\varepsilon \frac{\partial^{2} \underline{E}}{\partial t^{2}}=-\frac{1}{\mu} \nabla \times(\nabla \times \underline{E})
$$

However:

$$
\nabla \times(\nabla \times \underline{E})=\nabla(\nabla \cdot \underline{E})-\nabla^{2} \underline{E}
$$

It follows from equations (1.18), (1.19) and (1.14):

$$
\frac{\partial^{2} \underline{E}}{\partial t^{2}}=\frac{1}{\varepsilon \mu} \nabla^{2} \underline{E}=c^{2} \cdot \nabla^{2} \underline{E}
$$

Where $C$ is the speed of light.

For simplicity let's assume that the electromagnetic wave is planar and moves in the $\mathrm{x}$ direction. Since the partial derivatives with respect to $\mathrm{y}$ and $\mathrm{z}$ vanish we have:

$$
\frac{\partial^{2} \underline{E}}{\partial t^{2}}=c^{2} \cdot \frac{\partial^{2} \underline{E}}{\partial x^{2}}
$$

Equation (1.21) is the classical wave equation. The general solution to this equation is the following:

$$
\underline{E}(x, t)=a_{1} \cdot \underline{f}_{1}[k(x-c t)]+a_{2} \cdot \underline{f}_{2}[k(x+c t)]
$$

where $\underline{f}_{1}$ and $\underline{f}_{2}$ are any twice differentiable vector functions and $\boldsymbol{a}_{1}$ and $\boldsymbol{a}_{2}$ are two constant numbers. Since we are dealing with propagating waves $\underline{f}_{1}$ and $\underline{f}_{2}$ should be complex exponential vector functions. Therefore:

$$
\underline{E}(x, t)=\underline{v}_{1} \cdot e^{j[k(x-c t)]}+\underline{v}_{2} \cdot e^{j[k(x+c t)]}
$$

The solution (1.23) to equation (1.21) consists of an electromagnetic wave propagating in the positive direction of the $\mathrm{x}$ axis along with another wave in the opposite direction, both propagating at the nominal speed of light $\boldsymbol{c} . k=\frac{1}{\lambda}$, where $\lambda$ is the wavelength, and $k c=\omega$ is the frequency of the electromagnetic waves. $\underline{\boldsymbol{v}}_{1}$ and $\underline{\boldsymbol{v}}_{2}$ are constant complex vectors [2].

This is the classic solution of Maxwell's equation for a planar electromagnetic wave. As expected, the speed of propagation of the electromagnetic waves is the nominal speed of light $\boldsymbol{c}$ since there is no motion relative to the RCS (due to the restriction in the derivation of the common form of Maxwell's equations).

What happens when a radiation source moves with respect to the RCS? It follows from the assumption of the universal validity of Maxwell's equations (1.20) and (1.21) (namely: that they are valid in any inertial coordinate system) that the speed of propagation of any electromagnetic wave in all inertial coordinate systems is constant and equals to the nominal speed of light $c$ [solution (1.23) to equation (1.21)]. Thus, the speed of propagation of electromagnetic waves being constant in all inertial coordinate systems is not necessarily a measured observation. It is an assumption, a consequence of the assumed universal validity of the common Maxwell's equations even for dynamic systems.

Suppose that a radiation source moves at a speed $u$ in the positive direction of the $x$ axis of the RCS. As engineers (hopefully with some common sense), and in agreement with the Galilean transformation where velocity vectors are additive, we would expect the electric field vector, of the propagating planar electromagnetic wave parallel to the $\mathrm{x}$ axis, to have the following form with respect to the RCS:

$$
\underline{E}(x, t)=\underline{v}_{1} \cdot e^{j\{k[x-(c+u) t]\}}+\underline{v}_{2} \cdot e^{j\{k[x+(c-u) t]\}}
$$

Namely, we should have a wave propagating at a speed of $(\underline{c}+u)$ in the direction of the positive $\mathrm{x}$ axis and another wave propagating at a speed of $(c-u)$ in the direction of the negative $x$ axis. The electric field in equation (1.24) obviously does not comply with Maxwell's equation (1.21).

There are only three possibilities: Either equation (1.24) is wrong, or Maxwell's equation (1.21) is wrong, or both equations are wrong.

Lorentz assumed that equation (1.24) was wrong, which is equivalent to the assumption that the Galilean transformation does not apply, and went on to formulate his famous alternative transformation.

We will show that Maxwell's equation (1.21) is wrong since it is inadequate for dynamic systems. When the proper form of the Maxwell equations is applied - equation (1.24) is the right solution. This means that the Galilean transformation is valid (i.e.: the corrected Maxwell equations are invariant under the Galilean transformation). In addition, due to terms restored to the corrected Maxwell equations - they do not equate under the Lorentz transformation rendering this transformation, along with the theory of relativity which is based on it, invalid.

It is proven in the next chapter that when the corrected set of Maxwell equations is applied - the Galilean transformation is universally valid wherefrom it follows that Newton's laws of mechanics are universally valid and not just low speed approximations.

\section{Solution of the Corrected Maxwell Equations}

As noted in the previous chapter Maxwell's equations (1.10) to (1.12), along with their derivatives (1.20) and (1.21), were formulated for static systems, namely: no motion relative to the RCS. Their wrong application to dynamic systems led to the Lorentz transformation and Einstein's theory of relativity.

In this chapter a solution to the complete and corrected set of Maxwell equations [(1.1) to (1.8)] is presented. This solution demonstrates that the Galilean transformation and Newtonian physics are universally valid.

We proceed with the application of the corrected Maxwell equations to a planar wave in vacuum where all coordinate systems are inertial. It follows from the assumption that all coordinate systems, including the RCS, are inertial that the velocity vector $V$ in equations (1.1) and (1.2) is constant. Equations (1.1) and (1.2) become:

$$
\begin{aligned}
& \frac{\partial \underline{B}}{\partial t}+(\underline{V} \cdot \nabla) \underline{B}=-(\nabla \times \underline{E}) \text { Faraday's law } \\
& \frac{\partial \underline{D}}{\partial t}+(\underline{V} \cdot \nabla) \underline{D}=\nabla \times \underline{H} \text { Ampere's law }
\end{aligned}
$$


It follows from equations (1.7) and (1.8):

$\frac{\partial \underline{H}}{\partial t}+(\underline{V} \cdot \nabla) \underline{H}=\frac{-(\nabla \times \underline{E})}{\mu}$ Faraday's law

$\frac{\partial \underline{E}}{\partial t}+(\underline{V} \cdot \nabla) \underline{E}=\frac{\nabla \times \underline{H}}{\varepsilon}$ Ampere's law

It should be emphasized that there is a very big difference between the restoration of the missing terms to the corrected Maxwell equations [middle terms in equations [(2.1) to (2.4)] and the introduction of the Lorentz transformation. The Lorentz transformation was introduced to achieve a certain goal: avoiding inconsistencies which result from the wrong assumption that Maxwell's equations are universally valid. In contrast, the restoration of the missing terms to the corrected Maxwell equations is not done as a step in creating a new theory. It is a necessary step that follows from the correct derivation in appendix A of the differential equations corresponding to Faraday's and Ampere's laws. The omission of these terms has been a serious mistake.

Equations (2.3) and (2.4) can be rewritten as follows:

$$
\begin{aligned}
& \frac{D \underline{H}}{D t}=\frac{-(\nabla \times \underline{E})}{\mu} \text { Faraday's law } \\
& \frac{D \underline{E}}{D t}=\frac{\nabla \times \underline{H}}{\varepsilon} \text { Ampere's law }
\end{aligned}
$$

Where

$$
\frac{D}{D t}=\frac{d}{d t}=\frac{\partial}{\partial t}+(\underline{V} \cdot \nabla)
$$

is the total derivative, sometimes termed the convective derivative. As engineering students we often encountered the notion of the total derivative, especially in fluid dynamics. We were fortunate to have Professor David Pnueli Z"L as our instructor, an excellent teacher and a bright light in the darkness of the last two years in college.

Differentiating equation (2.6) with respect to time (note that $\underline{V}$ is constant!)

$$
\frac{D^{2} \underline{E}}{D t^{2}}=\frac{\frac{D(\nabla \times \underline{H})}{D t}}{\varepsilon}=\frac{\nabla \times \frac{D \underline{H}}{D t}}{\varepsilon}
$$

Substituting equation (2.5) into (2.8):

$$
\frac{D^{2} \underline{E}}{D t^{2}}=-\frac{\nabla \times(\nabla \times \underline{E})}{\varepsilon \mu}=-c^{2} \cdot \nabla \times(\nabla \times \underline{E})
$$

From equation (2.7):

$$
\frac{D^{2} \underline{E}}{D t^{2}}=\left[\frac{\partial}{\partial t}+(\underline{V} \cdot \nabla)\right]^{2} \underline{E}
$$

Using equation $(1.14)[(\nabla \cdot \underline{E})=0]$

$$
\nabla \times(\nabla \times \underline{E})=\nabla(\nabla \cdot \underline{E})-\nabla^{2} \underline{E}=-\nabla^{2} \underline{E}
$$

Substituting equations (2.10) and (2.11) into (2.9):

$$
\left[\frac{\partial}{\partial t}+(\underline{V} \cdot \nabla)\right]^{2} \underline{E}=c^{2} \cdot \nabla^{2} \underline{E}
$$

We assume again that the electromagnetic wave is planar and propagating along the $\mathrm{x}$ axis, in which case the derivatives with respect to $y$ and $z$ vanish:

$$
\underline{V}=\left[\begin{array}{c}
u \\
0 \\
0
\end{array}\right] ; \quad(\underline{V} \cdot \nabla) \underline{E}=u \frac{\partial \underline{E}}{\partial x}
$$

$$
\begin{aligned}
& {\left[\frac{\partial}{\partial t}+(\underline{V} \cdot \nabla)\right]^{2} \underline{E}=\frac{\partial^{2} \underline{E}}{\partial t^{2}}+2 u \frac{\partial^{2} \underline{E}}{\partial x \partial t}+u^{2} \frac{\partial^{2} \underline{E}}{\partial x^{2}}} \\
& c^{2} \cdot \nabla^{2} \underline{E}=c^{2} \frac{\partial^{2} \underline{E}}{\partial x^{2}}
\end{aligned}
$$

Substituting equations (2.14) and (2.15) into equation (2.12) we obtain:

$$
\frac{\partial^{2} \underline{E}}{\partial t^{2}}+2 u \frac{\partial^{2} \underline{E}}{\partial x \partial t}+u^{2} \frac{\partial^{2} \underline{E}}{\partial x^{2}}=c^{2} \frac{\partial^{2} \underline{E}}{\partial x^{2}}
$$

Rearranging equation (2.16) yields:

$$
\frac{\partial^{2} \underline{E}}{\partial t^{2}}+2 u \frac{\partial^{2} \underline{E}}{\partial x \partial t}=\left(c^{2}-u^{2}\right) \frac{\partial^{2} \underline{E}}{\partial x^{2}}
$$

The factor $\left(c^{2}-u^{2}\right)=c^{2}\left[1-\left(\frac{u}{c}\right)^{2}\right]=c^{2}\left(1-\beta^{2}\right)$ at the right hand side of equation (2.17) is reminiscent of the factor involved in time, length and mass changes in the theory of special relativity. However, the theory of special relativity is faulty due to wrongly concluding that time-rate is contracted by the factor $\sqrt{1-\beta^{2}}$, in addition to the omission of the middle term in equation (2.17). We proceed to solve equation (2.17) by adding the term $\xi c \frac{\partial^{2} \underline{E}}{\partial x \partial t}$ to
both sides of the equation:

$$
\frac{\partial}{\partial t}\left[\frac{\partial \underline{E}}{\partial t}+(2 u+\xi c) \frac{\partial \underline{E}}{\partial x}\right]=\xi_{c} \frac{\partial}{\partial x}\left[\frac{\partial \underline{E}}{\partial t}+\frac{c^{2}-u^{2}}{\xi_{c}} \frac{\partial \underline{E}}{\partial x}\right]
$$

In order to create a common factor on both sides of equation (2.18) we require that:

$$
2 u+\xi_{c}=\frac{c^{2}-u^{2}}{\xi_{c}}
$$

The solution of equation (2.19) for $\xi$ leads to the following quadratic equation:

$$
\xi^{2}+2 \frac{u}{c} \xi-\left[1-\left(\frac{u}{c}\right)^{2}\right]=0
$$

The solution of equation $(2.20)$ is:

$$
\xi_{1,2}=-\frac{u}{c} \pm \sqrt{\left(\frac{u}{c}\right)^{2}+1-\left(\frac{u}{c}\right)^{2}}=-\frac{u}{c} \pm 1
$$

Substituting either one of the values $\xi_{1,2}$ into equation (2.18) yields the same result:

$$
\left[\frac{\partial}{\partial t}-(c-u) \frac{\partial}{\partial x}\right]\left[\frac{\partial}{\partial t}+(c+u) \frac{\partial}{\partial x}\right] \underline{E}=0
$$

The general solution to the partial differential equation (2.22) is the following:

$$
\underline{E}(x, t)=a_{1} \cdot \underline{f}_{1}\{k[x-(c+u) t]\}+a_{2} \cdot \underline{f}_{2}\{k[x+(c-u) t]\}
$$

where $\underline{f}_{1}$ and $\underline{f}_{2}$ are any twice differentiable vector functions while $a_{1}$ and $a_{2}$ are two constant numbers. Since we are dealing with propagating wavesL $\underline{f}_{1}$ and $\underline{f}_{2}$ should be complex exponential vector functions. Therefore:

$$
\underline{E}(x, t)=\underline{v}_{1} \cdot e^{j\{k[x-(c+u) t]\}}+\underline{v}_{2} \cdot e^{j\{k[x+(c-u) t]\}}
$$

$k=\frac{1}{\lambda}$, where $\lambda$ is the wavelength, $k(c+u)=\omega_{f}$ is the frequency of the forward propagating electromagnetic wave and $k(c-u)=\omega_{b}$ is the frequency of the backward propagating electromagnetic wave, as viewed by a stationary observer with respect to the RCS. $\underline{v}_{1}$ and $\underline{v}_{2}$ are constant complex vectors. The above mentioned variation of the electromagnetic wave frequency is the manifestation of the Doppler Effect. 
Equation (2.24) represents two waves: one wave propagating forward at a speed of $(c+u)$ in the direction of the positive $\mathrm{x}$ axis and another wave propagating backward at a speed of $(c-u)$ in the direction of the negative $\mathrm{x}$ axis, both with respect to the RCS. Equation (2.24) is identical to equation (1.24), the solution which we should have arrived at by common sense.

The significance of equation (2.24) is that the Galilean transformation is valid. The Lorentz transformation and Einstein's theory of special relativity are faulty and we may safely and comfortably return to the Galilean transformation and Newtonian mechanics.

The "magnet and coil" problem is obviously resolved by the application of the corrected Maxwell equations, as well as the "Faraday paradox" in section A.8.

\section{Definitions}

Current laws: The current version of the Faraday, Ampere and Gauss electric and magnetic laws.

Current equations: The EM (Maxwell's) equations based on the Current Laws and the Lorentz transformation (i.e.: the current relativistic EM equations).
Corrected laws: A corrected version of the Current Laws so that the speed of propagation of electric

and magnetic fields does not exceed the speed of light (if they are ever formulated).

Corrected equations: The EM equations based on the Corrected Laws (and, possibly, on the Lorentz Transformation which are illustrated in Figure 1.

\section{Derivation of the Electromagnetic Equations Corre- sponding to Faraday's, Ampere's and Charge Conserva- tion Laws}

This appendix presents, among other things, the derivation of the complete (corrected) Maxwell equations which contain time derivatives, namely: Faraday's, Ampere's and the charge conservation laws. These derivations are based on chapters 3 and 4 of reference 1 which present Maxwell's equations in integral form and differential form, respectively.

Where: $\underline{D}=\varepsilon E$ and $\underline{H}=\mu \underline{B}$.

The following definition is needed for the subsequent derivations:

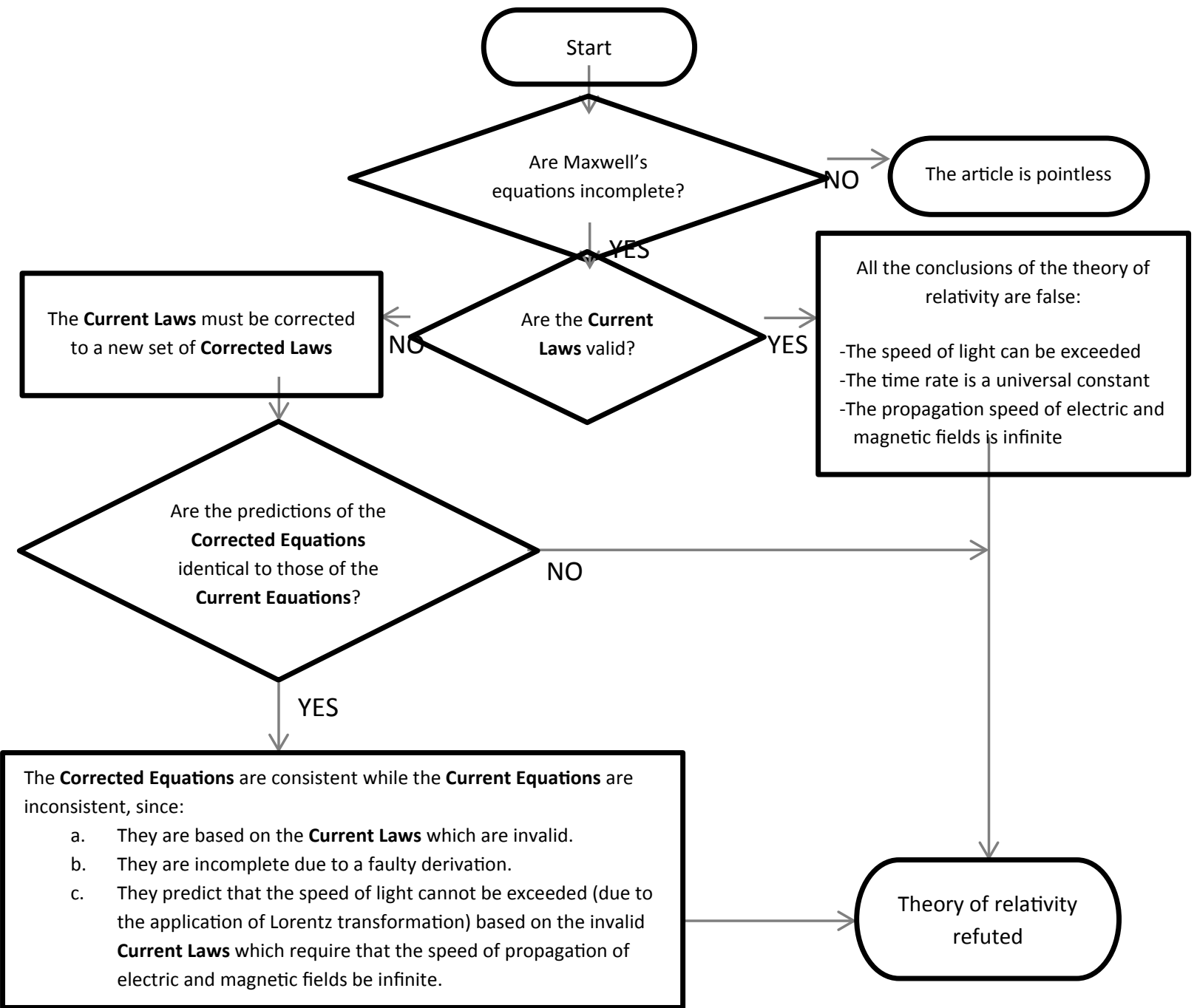

Figure 1: Illustration and comparison of standard laws 
An arbitrary surface $A$ with a closed boundary $S$ is selected. A pointer on $S$ indicating a sense of travel is provided, and the direction of the normal to the surface $A$ (the direction of an elemental area vector $\underline{d A}$ ) is defined as positive which forms a right handed screw with the $S$ pointer.

A detailed explanation of the following terms: the velocity vector field $\underline{V}$, the surface $A$ and the closed volume $\mathbf{v o l}$, is presented in the fifth paragraph of chapter 1 while discussing the notions of static and dynamic systems.

\section{Faraday's law}

Faraday's law in integral form is given in equation (A.1.1)

$\frac{d}{d t} \int_{A} \underline{B} \cdot \underline{d A}=-\oint_{s} \underline{E} \cdot \underline{d s}$

It follows from Stokes' theorem

$\frac{d}{d t} \int_{A} \underline{B} \cdot \underline{d A}=-\int_{A}(\nabla \times \underline{E}) \cdot \underline{d A}$

We proceed to evaluate the term $\frac{d}{d t} \int_{A} \underline{B} \cdot \underline{d A}$ in equation (A.1.2). A derivation of this expression is presented in reference 2, volume 2 page 346 .

$\frac{d}{d t} \int_{A} \underline{B} \cdot \underline{d A}=\lim _{\Delta t \rightarrow 0} \frac{\int_{A(t+\Delta t)} \underline{B}(t+\Delta t) \cdot \underline{d A}-\int_{A(t)} \underline{B}(t) \cdot \underline{d A}}{\Delta t}$

The above equation can be rewritten as follows:

$\frac{d}{d t} \int_{A} \underline{B} \cdot \underline{d A}=\lim _{\Delta t \rightarrow 0} \frac{\int_{A(t+\Delta t)} \underline{B}(t+\Delta t) \cdot \underline{d A}-\int_{A(t+\Delta t)} \underline{B}(t) \cdot \underline{d A}+\int_{A(t+\Delta t)} \underline{B}(t) \cdot \underline{d A}-\int_{A(t)} \underline{B}(t) \cdot \underline{d A}}{\Delta t}$

Hence:

$\frac{d}{d t} \int_{A} \underline{B} \cdot \underline{d A}=\int_{A} \lim _{\Delta t \rightarrow 0} \frac{[\underline{B}(t+\Delta t)-\underline{B}(t)]}{\Delta t} \cdot \underline{d A}+\lim _{\Delta t \rightarrow 0} \frac{\int_{A(t+\Delta t)} \underline{B}(t) \cdot \underline{d A}-\int_{A(t)} \underline{B}(t) \cdot \underline{d A}}{\Delta t}$

Therefore:

$\frac{d}{d t} \int_{A} \underline{B} \cdot \underline{d A}=\int_{A} \frac{\partial \underline{B}}{\partial t} \cdot \underline{d A}+\lim _{\Delta t \rightarrow 0} \frac{\int_{A(t+\Delta t)} \underline{B}(t) \cdot \underline{d A}-\int_{A(t)} \underline{B}(t) \cdot \underline{d A}}{\Delta t}$

To evaluate the last term in equation (A.1.3) we need some visualization, see Figure 2. Imagine the surface $A(t)$ as the bottom of a box and $A(t+t)$ as the top surface of that box. Each point on $A(t)$ is connected to a corresponding point on $A(t+\Delta t)$ by a vector $V \Delta t$ . The velocity vector $V$ may vary space-wise (in addition to timewise variation) so that the top surface $A(t+\Delta t)$ is in general rotated and deformed relative to $A(t)$. In Figure 2 some of the vectors $V \Delta t$ connecting the circumference of $A(t)$ to that of $A(t+t)$ are drawn,

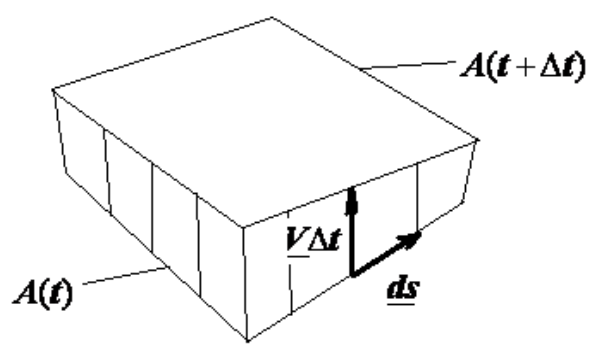

Figure 2: Visualization of the surface. thus constructing the side envelope of the above mentioned "box". We now perform the following integral over the entire outer surface of the box.

$$
\oint_{B o x} \underline{B}(t) \cdot \underline{d A}=\int_{A(t+\Delta t)} \underline{B}(t) \cdot \underline{d A}-\int_{A(t)} \underline{B}(t) \cdot \underline{d A}+\oint_{s} \underline{B}(t) \cdot(\underline{d s} \times \underline{V} \Delta t)
$$

The three terms at the right hand side of equation (A.1.4) correspond to the integrals over the top of the box, the bottom of the box and the side envelope, respectively. Note that the term $\underline{d s} \times \underline{V} \Delta t$ [in the line integral of equation (A.1.4)] is an elemental area vector $d A$ of the side envelope of the box. According to Gauss's theorem:

$$
\oint_{B o x} \underline{B}(t) \cdot \underline{d A}=\int_{V o l}(\nabla \cdot \underline{B}) d(V o l)=\int_{A(t)}(\nabla \cdot \underline{B}) \Delta t \underline{V} \cdot \underline{d A}
$$

$\mathrm{Vol}$ is the volume of the box and $d(\mathrm{Vol})=\Delta t \underline{\mathrm{V}} \cdot \underline{\mathrm{A} A}$ is an elemental volume of that "box". It follows from equations (A.1.4) and (A.1.5):

$\int_{A(t+\Delta t)} \underline{B}(t) \cdot \underline{d A}-\int_{A(t)} \underline{B}(t) \cdot \underline{d A}=\int_{A(t)}[\nabla \cdot \underline{B}(t)] \Delta t \underline{V} \cdot \underline{d A}-\oint_{s} \underline{B}(t) \cdot(\underline{d s} \times \underline{V} \Delta t)$

Substitution of equation (A.1.6) into (A.1.3) and realizing that $\underline{B} \cdot(\underline{d s} \times \underline{V})=(\underline{V} \times \underline{B}) \cdot \underline{d s}$, yields:

$\frac{d}{d t} \int_{A} \underline{B} \cdot \underline{d A}=\int_{A} \frac{\partial \underline{B}}{\partial t} \cdot \underline{d A}+\int_{A}(\nabla \cdot \underline{B}) \underline{V} \cdot \underline{d A}-\oint_{s}(\underline{V} \times \underline{B}) \cdot \underline{d s}$

From equations (A.1.7) and (A.1.2) we obtain:

$\int_{A} \frac{\partial \underline{B}}{\partial t} \cdot \underline{d A}+\int_{A}(\nabla \cdot \underline{B}) \underline{V} \cdot \underline{d A}-\oint_{s}(\underline{V} \times \underline{B}) \cdot \underline{d s}=-\int_{A}(\nabla \times \underline{E}) \cdot \underline{d A}$

But according to Stoke's theorem:

$\oint_{s}(\underline{V} \times \underline{B}) \cdot \underline{d s}=\int_{A}[\nabla \times(\underline{V} \times \underline{B})] \cdot \underline{d A}$

Substitution of the previous equation into equation (A.1.8) yields:

$\int_{A}\left[\frac{\partial \underline{B}}{\partial t}+(\nabla \cdot \underline{B}) \underline{V}-\nabla \times(\underline{V} \times \underline{B})+(\nabla \times \underline{E})\right] \cdot \underline{d A}=0$

Since equation (A.1.9) is valid for any elemental area $A$ :

$\frac{\partial \underline{B}}{\partial t}+(\nabla \cdot \underline{B}) \underline{V}-\nabla \times(\underline{V} \times \underline{B})=-(\nabla \times \underline{E})$

Using the vector identity

$\nabla \times(\underline{V} \times \underline{B}) \equiv(\nabla \cdot \underline{B}+\underline{B} \cdot \nabla) \underline{V}-(\nabla \cdot \underline{V}+\underline{V} \cdot \nabla) \underline{B}$

equation (A.1.10) may be rewritten as follows:

$\frac{\partial \underline{B}}{\partial t}+(\underline{V} \cdot \nabla) \underline{B}=-(\nabla \times \underline{E})+[(\underline{B} \cdot \nabla) \underline{V}-(\nabla \cdot \underline{V}) \underline{B}]$

Since $\nabla \cdot \underline{B}=0$, according to Gauss's magnetic flux law [equation (1.3)], an alternative form of the differential equation corresponding to Faraday's law is obtained from equation (A.1.10).

$\frac{\partial \underline{B}}{\partial t}-\nabla \times(\underline{V} \times \underline{B})=-(\nabla \times \underline{E})$

Equations (A.1.11) and (A.1.12) are two equivalent most general forms of the corrected Maxwell equation corresponding to Faraday's law. If the velocity vector $V$ does not vary space-wise, in particular when $\underline{V}$ is constant, the last two terms in equation (A.1.11) vanish and the corrected Maxwell equation corresponding to Faraday's law assumes the following form:

$\frac{\partial \underline{B}}{\partial t}+(\underline{V} \cdot \nabla) \underline{B}=-(\nabla \times \underline{E})$ 


\section{Ampere's law}

Ampere's law in integral form is presented in equation (A.2.1).

$\frac{d}{d t} \int_{A} \underline{D} \cdot \underline{d A}+\int_{A} \underline{J} \cdot \underline{d A}=\oint_{s} \underline{H} \cdot \underline{d s}$

It follows from Stokes' theorem:

$\frac{d}{d t} \int_{A} \underline{D} \cdot \underline{d A}+\int_{A} \underline{J} \cdot \underline{d A}=\int_{A}(\nabla \times \underline{H}) \cdot \underline{d A}$

From equation (A.1.7) we obtain, by replacing $\underline{B}$ by $\underline{D}$ :

$\frac{d}{d t} \int_{A} \underline{D} \cdot \underline{d A}=\int_{A} \frac{\partial \underline{D}}{\partial t} \cdot \underline{d A}+\int_{A}(\nabla \cdot \underline{D}) \underline{V} \cdot \underline{d A}-\oint_{s}(\underline{V} \times \underline{D}) \cdot \underline{d s}$

From equations (A.2.2) and (A.2.3) it follows:

$\int_{A} \frac{\partial \underline{D}}{\partial t} \cdot \underline{d A}+\int_{A}(\nabla \cdot \underline{D}) \underline{V} \cdot \underline{d A}-\oint_{s}(\underline{V} \times \underline{D}) \cdot \underline{d S}+\int_{A} \underline{J} \cdot \underline{d A}=\int_{A}(\nabla \times \underline{H}) \cdot \underline{d A}$

But according to Stoke's theorem:

$\oint_{s}(\underline{V} \times \underline{D}) \cdot \underline{d s}=\int_{A}[\nabla \times(\underline{V} \times \underline{D})] \cdot \underline{d A}$

Substitution of the previous equation into equation (A.2.4) yields:

$\int_{A}\left[\frac{\partial \underline{D}}{\partial t}+(\nabla \cdot \underline{D}) \underline{V}-\nabla \times(\underline{V} \times \underline{D})+\underline{J}-(\nabla \times \underline{H})\right] \cdot \underline{d A}=0$

Since equation (A.2.5) is valid for any elemental area $A$ :

$\frac{\partial \underline{D}}{\partial t}+(\nabla \cdot \underline{D}) \underline{V}-\nabla \times(\underline{V} \times \underline{D})+\underline{J}=(\nabla \times \underline{H})$

Using the vector identity

$\nabla \times(\underline{V} \times \underline{D}) \equiv(\nabla \cdot \underline{D}+\underline{D} \cdot \nabla) \underline{V}-(\nabla \cdot \underline{V}+\underline{V} \cdot \nabla) \underline{D}$

equation (A.2.6) may be rewritten as follows:

$\frac{\partial \underline{D}}{\partial t}+(\underline{V} \cdot \nabla) \underline{D}+\underline{J}=(\nabla \times \underline{H})+[(\underline{D} \cdot \nabla) \underline{V}-(\nabla \cdot \underline{V}) \underline{D}]$

Since $\nabla \cdot D=\rho$, according to Gauss's electric flux law [equation (1.4)], an alternative form of the differential equation corresponding to Faraday's law is obtained from equation (A.2.6).

$$
\frac{\partial \underline{D}}{\partial t}-\nabla \times(\underline{V} \times \underline{D})+\rho \underline{V}+\underline{J}=(\nabla \times \underline{H})
$$

Equation (A.2.7) and (A.2.8) are two equivalent most general forms of the corrected Maxwell equation corresponding to Ampere's law. If the velocity vector $\underline{V}$ does not vary space-wise, in particular when $\underline{V}$ is constant, the last two terms in equation (A.2.7) vanish and the corrected Maxwell equation corresponding to Ampere's law assumes the following form:

$$
\frac{\partial \underline{D}}{\partial t}+(\underline{V} \cdot \nabla) \underline{D}+\underline{J}=(\nabla \times \underline{H})
$$

\section{Charge Conservation Law}

The charge conservation law states that for any control volume, whether stationary or moving, the rate of change of total charge plus the charge leaving by conduction currents (through the surface enclosing the control volume) equals zero. It is assumed that no charge is created, i.e.: no nuclear reactions.

An arbitrary volume $v o l$ is selected which is enclosed in a surface $A$.

$$
\frac{d}{d t} \int_{V o l} \rho d(V o l)+\oint_{A} \underline{J} \cdot \underline{d A}=0
$$

We first compute the left term in equation (A.3.1) applying a method similar to the one employed in section A.1 dealing with Faraday's law.

$$
\begin{array}{r}
\frac{d}{d t} \int_{V o l} \rho d(V o l)=\lim _{\Delta t \rightarrow 0} \frac{1}{\Delta t}\left[\int_{V o l(t+\Delta t)} \rho(t+\Delta t) d(V o l)-\int_{V o l(t)} \rho(t) d(V o l)\right]= \\
\lim _{\Delta t \rightarrow 0} \frac{1}{\Delta t}[ \\
{\left[\int_{V o l(t+\Delta t)} \rho(t+\Delta t) d(V o l)-\int_{V o l(t+\Delta t)} \rho(t) d(V o l)+\right.} \\
\left.\int_{V o l(t+\Delta t)} \rho(t) d(V o l)-\int_{V o l(t)} \rho(t) d(V o l)\right]
\end{array}
$$

It follows from equation (A.3.2):

$$
\begin{aligned}
\frac{d}{d t} \int_{V o l} \rho d(V o l)= & \lim _{\Delta t \rightarrow 0} \frac{1}{\Delta t}\left[\int_{V o l(t+\Delta t)} \rho(t) d(V o l)-\int_{V o l(t)} \rho(t) d(V o l)\right]+ \\
& \int_{V o l} \frac{\partial \rho}{\partial t} d(V o l)
\end{aligned}
$$

The limit at the right hand side of equation (A.3.3) is the rate of charge flowing out of the control volume through the surface $A$. Note that it is not a conduction current, but rather a "convection" current.

$\frac{d}{d t} \int_{V o l} \rho d(V o l)=\oint_{A} \rho \underline{V} \cdot \underline{d A}+\int_{V o l} \frac{\partial \rho}{\partial t} d(V o l)$

Substitution of equation (A.3.4) into equation (A.3.1) yield:

$\int_{V o l} \frac{\partial \rho}{\partial t} d(V o l)+\oint_{A} \rho \underline{V} \cdot \underline{d A}+\oint_{A} \underline{J} \cdot \underline{d A}=0$

Applying Gauss's theorem to equation (A.3.5) we obtain:

$\int_{V o l}\left[\frac{\partial \rho}{\partial t}+\nabla \cdot(\rho \underline{V})+\nabla \cdot \underline{J}\right] d(V o l)=0$

Since equation (A.3.6) is valid for any volume vol - the integrand must vanish. Therefore:

$\frac{\partial \rho}{\partial t}+\nabla \cdot(\rho \underline{V})+\nabla \cdot \underline{J}=0$

Equation (A.3.7) is the corrected Maxwell equation corresponding to the charge conservation law.

Using the identity $\nabla \cdot(\rho \underline{V}) \equiv(\underline{V} \cdot \nabla) \rho+\rho(\nabla \cdot \underline{V})$ equation (A.3.7) may be rewritten as follows:

$$
\frac{\partial \rho}{\partial t}+(\underline{V} \cdot \nabla) \rho+\rho(\nabla \cdot \underline{V})+\nabla \cdot \underline{J}=0
$$

\section{Summary. The Lorentz Field and a Similar Magnetic Field}

To summarize, we write down again the corrected Maxwell's equations corresponding to Faraday's, Ampere's and charge conservation laws.

From equations (A.1.11), (A.1.12), (A.2.7) and (A.3.8):

$\frac{\partial \underline{B}}{\partial t}+(\underline{V} \cdot \nabla) \underline{B}=-(\nabla \times \underline{E})+[(\underline{B} \cdot \nabla) \underline{V}-(\nabla \cdot \underline{V}) \underline{B}]$ Faraday's law (A.4.1)

$\frac{\partial \underline{B}}{\partial t}-\nabla \times(\underline{V} \times \underline{B})=-(\nabla \times \underline{E})$ Equivalent Faraday's law

$\frac{\partial \underline{D}}{\partial t}+(\underline{V} \cdot \nabla) \underline{D}+\underline{J}=(\nabla \times \underline{H})+[(\underline{D} \cdot \nabla) \underline{V}-(\nabla \cdot \underline{V}) \underline{D}]$ Ampere's law (A.4.3)

$\frac{\partial \underline{D}}{\partial t}-\nabla \times(\underline{V} \times \underline{D})+\rho \underline{V}+\underline{J}=(\nabla \times \underline{H})$ Equivalent Ampere's law

$\frac{\partial \rho}{\partial t}+(\underline{V} \cdot \nabla) \rho+\rho(\nabla \cdot V)+\nabla \cdot \underline{J}=0$ Charge conservation law

The total time derivative of any vector field $\underline{W}$ may be expanded as follows: 
$\frac{d \underline{W}[t, \underline{r}(t)]}{d t}=\frac{\partial \underline{W}}{\partial t}+\frac{\partial \underline{W}}{\partial r} \cdot \frac{d \underline{r}}{d t}=\frac{\partial \underline{W}}{\partial t}+\frac{\partial \underline{W}}{\partial r} \cdot \underline{V}=\frac{\partial \underline{W}}{\partial t}+(\underline{V} \cdot \nabla) \underline{W}$

Equation (A.4.6) is valid for scalar fields as well.

Applying equation (A.4.6) allows rewriting equations (A.4.1), (A.4.3) and (A.4.4):

$\frac{d \underline{B}}{d t}=-(\nabla \times \underline{E})+[(\underline{B} \cdot \nabla) \underline{V}-(\nabla \cdot \underline{V}) \underline{B}]$ Faraday's law

$\frac{d \underline{D}}{d t}+\underline{J}=(\nabla \times \underline{H})+[(\underline{D} \cdot \nabla) \underline{V}-(\nabla \cdot \underline{V}) \underline{D}]$ Ampere's law

$\frac{d \rho}{d t}+\rho(\nabla \cdot \underline{V})+\nabla \cdot \underline{J}=0$ Charge conservation law

When the velocity vector $V$ does not vary space-wise, in particular when $\underline{V}$ is constant, equations (A.4.7) to (A.4.9) become:

$$
\begin{aligned}
& \frac{d \underline{B}}{d t}=\frac{\partial \underline{B}}{\partial t}+(\underline{V} \cdot \nabla) \underline{B}=-(\nabla \times \underline{E}) \text { Faraday's law } \\
& \frac{d \underline{D}}{d t}+\underline{J}=\frac{\partial \underline{D}}{\partial t}+(\underline{V} \cdot \nabla) \underline{D}+\underline{J}=(\nabla \times \underline{H}) \text { Ampere's law } \\
& \frac{d \rho}{d t}+\nabla \cdot \underline{J}=\frac{\partial \rho}{\partial t}+(\underline{V} \cdot \nabla) \rho+\nabla \cdot \underline{J}=0 \text { Charge conservation law }
\end{aligned}
$$

In the very special case where $V=0$, which means that there is no motion relative to the RCS (in other words, the system is static and the volume vol and surface $A$ are at rest), equations (A.4.10) to (A.4.12) become:

$$
\begin{aligned}
& \frac{\partial \underline{B}}{-}=-(\nabla \times E) \text { Faraday's law } \\
& \frac{\partial \underline{D}}{\partial t}+\underline{J}=(\nabla \times \underline{H}) \text { Ampere's law } \\
& \frac{\partial \rho}{\partial t}+\nabla \cdot \underline{J}=0 \text { Charge conservation law }
\end{aligned}
$$

The last three equations are the common formulations of Ampere's, Faraday's and charge conservation laws in Maxwell's equations. This is a very important point that should be emphasized: The common Maxwell's equations are valid only for systems at rest (i.e.: static systems, $\underline{V}=0)$. The application of these equations to dynamic systems, where $\bar{V} \neq 0$, (often termed "the universal validity of Maxwell's equations") is the basis for the erroneous theory of relativity.

From the equivalent Faraday's law, equation (A.4.2), in steady-state conditions (where the partial time derivatives vanish) we obtain:

$$
\nabla \times(\underline{V} \times \underline{B})=\nabla \times \underline{E}
$$

Since $\nabla \times(\nabla \varphi)=0$ for any scalar field $\varphi$, we have from equation (A.4.16):

$$
\underline{E}=\underline{V} \times \underline{B}-\nabla \varphi
$$

The term $V \times B$ in equation (A.4.17) is the "Lorentz field", which yields the Lorentz force when multiplied by an electric charge. This field is relevant to many electromagnetic problems, one of which being the motion of charged particles in magnetic fields. The separate introduction of this field was necessary due to the fact that some electromagnetic phenomena could not be explained based solely on Maxwell's equations. It follows from equation (A.4.17) that the Lorentz field $\underline{E}_{L}=\underline{V} \times \underline{B}$ is an immediate consequence of the corrected
Maxwell equations, as a result of restoring the terms that are missing in the common representation of Maxwell's equations.

Similarly, when applying the equivalent Ampere's law, equation (A.4.4), in vacuum $(\rho=0$ and $\underline{J}=0)$ we obtain in steady-state conditions:

$$
\underline{H}=-(\underline{V} \times \underline{D})-\nabla \varphi
$$

Where $\varphi$ is again any scalar field. The contribution of the term $-(V \times \underline{D})$ to the magnetic excitation vector $\underline{H}$ in equation (A.4.18) is similar to the contribution of the Lorentz field $\underline{E}_{L}=\underline{V} \times \underline{B}$ to the electric field $\underline{E}$ in equation (A.4.17).

\section{Speed of Propagation of Electric and Magnetic Fields}

In this section we will prove that the physical laws on which Maxwell's equations are based imply that electric and magnetic fields propagate at an infinite speed. In other words, if the speed of propagation of electric and magnetic fields is finite - the following laws are inconsistent, i.e., they are self-contradictory.

The following four equations are Ampere's law, Faraday's law, Gauss's electricity flux law and Gauss's magnetic flux law, respectively, in integral form.

$$
\begin{aligned}
& \frac{d}{d t} \int_{A} \underline{D} \cdot \underline{d A}+\int_{A} \underline{J} \cdot \underline{d A}=\oint_{s} \underline{H} \cdot \underline{d s} \text { Ampere's law } \\
& \frac{d}{d t} \int_{A} \underline{B} \cdot \underline{d A}=-\oint_{s} \underline{E} \cdot \underline{d s} \text { Faraday's law } \\
& \oint_{A} \underline{D} \cdot \underline{d A}=4 \pi Q \text { Gauss's electricity flux law }
\end{aligned}
$$

$\oint_{A} \underline{B} \cdot \underline{d A}=0$ Gauss's magnetic flux law (A.5.4) Equations (A.5.1) and (A.5.2) are identical to equations (A.2.1) and (A.1.1), respectively. The definitions of the surface $A$ and the closed boundary $S$ are presented in the two paragraphs preceding equation (A.1.1). $Q$ is the total electric charge within the closed surface $A$ in equation (A.5.3).

The laws in equations (A.5.1) to (A.5.4) are universal and valid at all times, not just at steady state conditions. In the following paragraphs it is shown that these laws clearly imply an infinite propagation speed of electric and magnetic fields, contrary to a corollary of the theory of relativity that nothing can propagate at a speed greater than the speed of light.

Ampere's law in equation (A.5.1) states that if a current $i$ starts flowing in a straight wire perpendicular to a plane - a magnetic excitation vector $H$ instantaneously appears on any circle located axisymmetrically around the wire. This vector is tangent to the circle and its magnitude is $H=i /(2 \pi r), r$ being the radius of the circle. As this is true for any finite radius $r$, the speed of propagation of the magnetic excitation vector $\underline{H}$ must be infinite. Since the magnetic induction field vector $\underline{B}=\mu \underline{H}$ - its speed of propagation is infinite as well.

A similar argument can be used for Faraday's law in equation (A.5.2). If a magnetic flux $\varphi=\int_{A} \underline{B} \cdot \underline{d A}$ is time varying in a coil - an electric field vector $\underline{E}$ appears instantaneously on any circle located axi-symmetrically around the coil. This vector is tangent to the circle and its magnitude is $E=(d \varphi / d t) /(2 \pi r)$, where $r$ is the radius of the circle. As this is true for any finite radius $r$ - the speed of propagation of the electric field vector $\underline{E}$ must be infinite. Likewise for the electric excitation vector $\underline{D}=\varepsilon \underline{E}$. 
The following paragraphs expand on the proofs presented in the former two paragraphs.

Ampere's law is presented in equation (A.5.1). Figure 3 consists of two concentric circles connected by two adjacent lines. A long straight conductor is located at the center of the two circles and is perpendicular to the page. At $t=0$ ( $t$ stands for time) a current $i$ starts flowing in the wire at which time the integral on the left hand side of equation (A.5.1) changes from 0 to $i$. If the speed of propagation of the magnetic excitation vector $\underline{H}$ is finite - the line integral on the right side of equation (A.5.1) will change from 0 to $i$ at $t=t_{1}$ on the inner circle and at $t=t_{2}$ on the outer circle, where $t_{2}>t_{1}$. At any time $t_{1}<t<t_{2}$ the line integral $\oint \underline{H} \cdot \underline{d s}$ over the two circles, including the two adjacent straight lines, will equal $i$ while the total current flowing through the area between the two circles is 0. This is a clear violation of Ampere's law. The above mentioned violation is a corollary of the assumption that the speed of propagation is finite. Consequently, this assumption is proven wrong and the speed of propagation of the Magnetic excitation vector $\underline{H}$ must be infinite.

Faraday's law states that the integrals on both sides of equation (A.5.2) are equal for all surfaces $A$ with a common boundary $S$. Figure 4 is a cross section of a spherical surface and a circular plane (represented in the figure by the vertical line DU). The center of the spherical surface is o. The intersection between the spherical surface and the circular plane is a circle. This circle is the common boundary of the spherical surface and the circular plane. The horizontal line represents a coil that

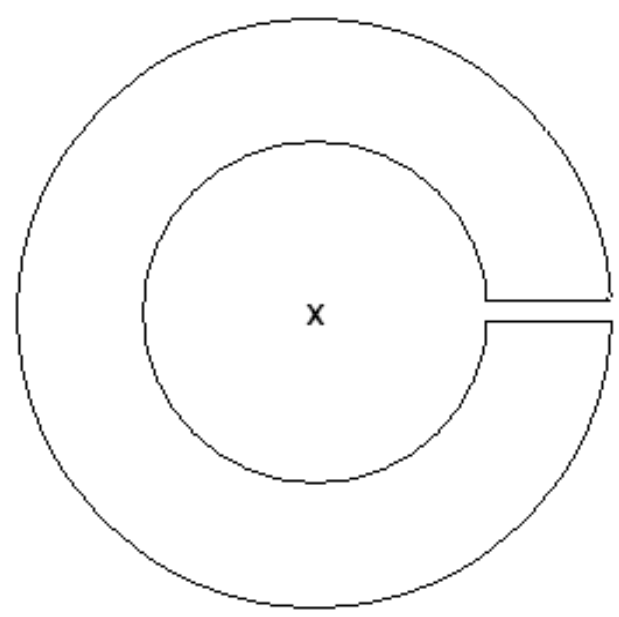

Figure 3: Two concentric circles connected by two adjacent lines.

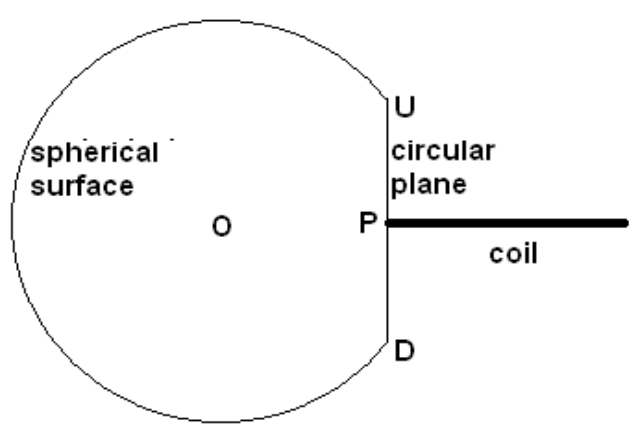

Figure 4: Cross section of a spherical surface and a circular plane. touches the circular plane at its center $P$. At $t=0$ a DC voltage is applied to the coil which causes the left hand side of equation (A.5.2) for the circular plane to change immediately from 0 to some nonzero value (since the coil touches the circular plane).

If the speed of propagation of the magnetic induction field vector $\underline{B}$ is finite - it will take a time span $t_{3}>0$ for all the lines of the vector field $\underline{B}$ to cross the spherical surface. For any time $0<t<t_{3}$ the left hand side term of equation (A.5.2) for the spherical surface will not equal that of the circular surface, which means that the value of the line integral on the right side of that equation is not unique. This is a violation of Faraday's law. The above mentioned violation is a corollary of the assumption that the speed of propagation is finite. Consequently, this assumption is proven wrong and the speed of propagation of the magnetic induction field vector $\underline{B}$ must be infinite.

Concerning Gauss's electricity flux law in equation (A.5.3) - Figure 5 presents a cross section of a sphere. This sphere is located in the upper atmosphere with a nitrogen atom at its center $\mathrm{o}$. At $t=0$ a cosmic ray hits the nucleus of the nitrogen atom and converts one of its protons to a neutron thus creating a carbon-14 atom. The number of electrons of this atom exceeds the number of protons by one, thus creating a charged particle at the center of the sphere.

If the speed of propagation of the electric excitation vector $\underline{D}$ is finite - the time it takes it to intersect the sphere in Figure 5 is $t_{4}>0$ For any time $0<t<t_{4}$ the integral at the left hand side of equation (A.5.2) equals 0 while the right hand side value of the same equation is $4 \pi e$, where $e$ is the charge of an electron. This is a clear violation of Gauss's electricity flux law. The above mentioned violation is a corollary of the assumption that the speed of propagation is finite. Consequently, this assumption is proven wrong and the speed of propagation of the electric excitation vector $\underline{D}$ must be infinite.

Concerning Gauss's magnetic flux law in equation (A.5.4) - Figure 6 presents a sphere being "illuminated" by a uniform magnetic induction vector field $\underline{B}$ from right to left. The "illuminating" electromagnet is turned on at $t=0$.

If the propagation speed of the magnetic induction vector field $B$ is finite - then at a certain time $t=t_{5}$ the front of this vector field will arrive at the center of the sphere. At that instant the integral at the left hand side of equation (A.5.4) will obviously not equal 0 , a clear violation of

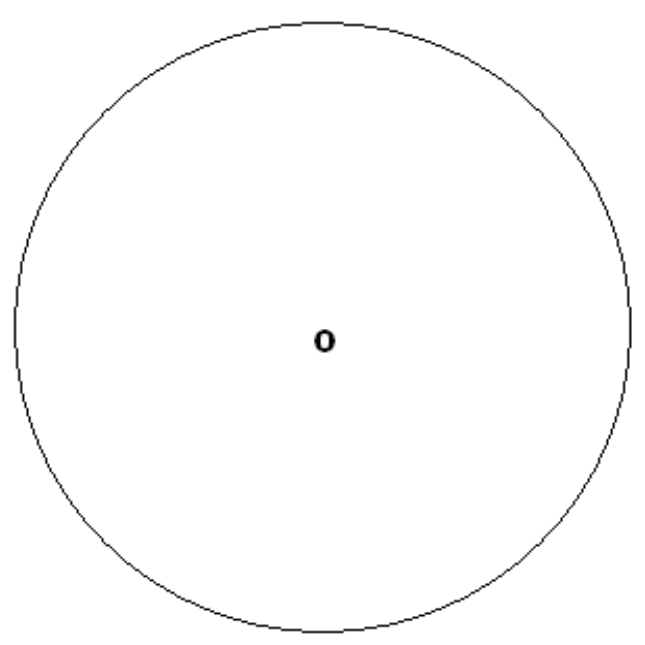

Figure 5: Cross section of a sphere. 
Gauss's magnetic flux law. The above mentioned violation is a corollary of the assumption that the speed of propagation is finite. Consequently, this assumption is proven wrong and the propagation speed of the magnetic induction vector field $\underline{B}$ must be infinite.

The last argument (concerning Gauss's magnetic flux law and Figure 6) and the second before last (Faraday's law and Figure 4) were seemingly disproved by professor Moshe Einat, in the name of professor Vladimir Bratman, both of the Ariel University, Ariel Israel. They pointed out that magnetic fields always consist of closed lines, and that during their propagation these lines inflate like a balloon. Therefore, the field lines never possess open ends - thus yielding the two above mentioned arguments invalid. However, this contention can also be rejected as follows: We place a round axisymmetric magnet rod in vacuum where there is neither conduction nor displacement currents. We then turn the magnet rod around its center point by 180 degrees thus swapping the location of its poles. The final magnetic field lines coincide with the initial lines while their direction is reversed. If the propagation speed of the magnetic field is finite - there exist three regions: The final region, the intermediate region (which corresponds to the time interval during which the magnet is rotated) and the initial region. The first two regions spread at the speed of light into the initial region.

We first prove that the vector $\underline{E}$ vanishes in the intermediate region. The differential equation that determines the electric field in that region is equation (1.20), namely: $\partial^{2} E / \partial t^{2}=c^{2} \cdot \nabla^{2} E$. Since the vector $\underline{E}$ vanishes in the initial and final regions $-E=0$ is a solution to the above differential equation. Since the solution to the EM equations is unique (as is proven in section A.7) - the electric vector field $\underline{E}$ vanishes in the intermediate region, along with $\underline{D}=\varepsilon \underline{E}$.

We freeze the picture of the magnetic field at a certain time and select a closed line consisting of four segments: Two of them are located at the final and initial regions along magnetic field lines and being connected by the other two segments which are always normal to field lines. Since the magnetic fields on the first two above mentioned segments point in opposite directions - the line integrals over them have the same sign and add up in absolute value. The line integrals over the other two segments vanish since they are orthogonal to the field lines. Therefore, the integral of the magnetic field along the above defined closed contour does not equal zero in spite the fact that $\underline{J}=0$ (vacuum) and $\underline{D}=0$ (as was proven in the previous paragraph), clearly violating Ampere's law in equation (A.5.1). The above mentioned violation is a corollary of the assumption that the speed of propagation is finite. Consequently, this assumption is proven wrong and the propagation speed of the magnetic field must be infinite.

Maxwell's equations, as well as the corrected Maxwell equations, are based on the integral laws in equations (A.5.1) to (A.5.4). Arriving at a conclusion that any speed cannot exceed the speed of light, on the basis of the above four integral laws which imply an infinite speed of propagation of electric and magnetic fields, does not make sense. If it is ever proven that the speed of light cannot be exceeded, then the above mentioned four laws will have to be modified. As a matter of fact - through a simple laboratory experiment it can be checked whether Faraday's law in equation (A.5.2) is correct, including the aspect of the infinite speed of propagation. However, it should be carefully planned since it involves ultra-high frequency electric signals.

\section{The Correct Version of Ampere's Law}

Several forms or Ampere's law can be found in the literature. One of them is equation (A.5.1) which is presented here as equation (A.6.1). $\frac{d}{d t} \int_{A} \underline{D} \cdot \underline{d A}+\int_{A} \underline{J} \cdot \underline{d A}=\oint_{s} \underline{H} \cdot \underline{d s}$

Two more versions Ampere's law are the following:

$\int_{A}\left(\frac{d \underline{D}}{d t}+\underline{J}\right) \cdot \underline{d A}=\oint_{s} \underline{H} \cdot \underline{d s}$

$\int_{A}\left(\frac{\partial \underline{D}}{\partial t}+\underline{J}\right) \cdot \underline{d A}=\oint_{s} \underline{H} \cdot \underline{d s}$

The last two equations are based on the premise that $\frac{d \underline{D}}{d t}$ is the "displacement current" which is added to the conduction current $J$ to obtain the total current.

Ampere's law as expressed in equation (A.6.3) is obviously wrong since it is limited to static cases only. Therefore, we have to determine whether equation (A.6.1) is the correct form of Ampere's law or equation (A.6.2).

Figure 7 is a cross section of a spherical surface $A$ intersected by a circular plane (represented in the figure by the vertical line DU). The center of the spherical surface is $\mathbf{o}$ at which point a charge $Q$ is located. The intersection between the spherical surface and the circular plane is a circle $S$ which is the boundary of the spherical surface $A$. The point $P$ is the center of the circular plane.

We now move the point $\mathrm{P}$ away from o until the circular boundary $S$ converges to a point. The integral on the right hand side of equations (A.6.1) to (A.6.3) converges to zero since the range of integration vanishes Figure 7 . Thus, in this particular case equation (A.6.1) becomes:

$$
\frac{d}{d t} \oint_{A} \underline{D} \cdot \underline{d A}+\oint_{A} \underline{J} \cdot \underline{d A}=0
$$

Similarly, equation (A.6.2) turns into:

$$
\oint_{A}\left(\frac{d \underline{D}}{d t}+\underline{J}\right) \cdot \underline{d A}=0
$$

We make two further assumptions. The first is that the whole setup is located in vacuum, where the conductivity is zero, thus the conduction current $J$ vanishes. Therefore we are left with a closed sphere and a charge $Q>0$ at its center. The second assumption is that the radius $r$ of the sphere is shrinking at rate of $\frac{d r}{d t}=-v$.

In this case Ampere's law in equation (A.6.4) should satisfy:

$$
\frac{d}{d t} \oint_{A} \underline{D} \cdot \underline{d A}=0
$$

And according equation (A.6.5) Ampere's law should be:

$\oint_{A} \frac{d \underline{D}}{d t} \cdot \underline{d A}=0$

Due to symmetry - the electric excitation vector $\underline{D}$ is normal to the spherical surface at all points and its magnitude $D$ equals:

$$
D=|\underline{D}|=\frac{Q}{4 \pi r^{2}}
$$

It follows from equation (A.6.8):

$$
\frac{d D}{d t}=\frac{\partial D}{\partial r} \frac{d r}{d t}=\left(-\frac{Q}{2 \pi r^{3}}\right)(-v)=\frac{Q v}{2 \pi r^{3}}
$$

We compute the integral $\oint_{A} \frac{d \underline{D}}{d t} \cdot \underline{d A}$ in equation (A.6.7). Again, due
inmetry: to symmetry:

$$
\oint_{A} \frac{d \underline{D}}{d t} \cdot \underline{d A}=\frac{d D}{d t} 4 \pi r^{2}=\frac{Q v}{2 \pi r^{3}} 4 \pi r^{2}=\frac{2 Q v}{r} \neq 0
$$




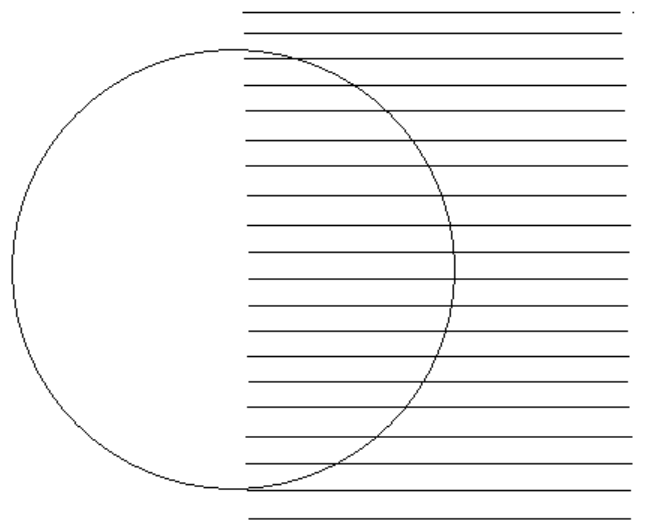

Figure 6: Sphere being "illuminated" by a uniform magnetic induction vector field from right to left.

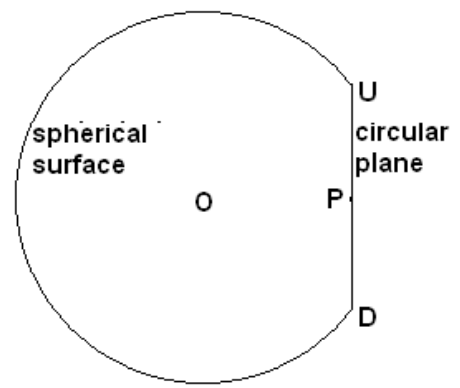

Figure 7: Cross section of a spherical surface $A$ intersected by a circular plane.

Equation (A.6.10) clearly contradicts the requirement of equation (A.6.7), which means that equation (A.6.2) is not a correct version of Ampere's law.

We compute the integral $\frac{d}{d t} \oint_{A} \underline{D} \cdot \underline{d A}$ in equation (A.6.6). In view of equation (A.6.8) and making use of symmetry again:

$$
\frac{d}{d t} \oint_{A} \underline{D} \cdot \underline{d A}=\frac{d}{d t}\left(D \cdot 4 \pi r^{2}\right)=\frac{d}{d t}(Q)=0
$$

The requirement of equation (A.6.6) is fulfilled; hence equation (A.6.1) is the correct form of Ampere's law.

\section{Existence and Uniqueness of Solutions to the EM Equations}

A necessary condition for the corrected Maxwell equations to be a well-defined mathematical problem (in other words: a requirement that these equations have a unique solution) is the equality between the number of independent equations and the number of variables. If the number of variables exceeds the number of independent equations - the solution is not unique. If the number of independent equations exceeds the number of variables - there is no solution.

From equations (1.1) to (1.8) we can determine the number of variables and equations.

The variables are the following: $\underline{B}, \underline{D}, \underline{E}, \underline{H}, \underline{J}$ and $\rho$. We have five vector variables and one scalar variable. Each vector variable consists of three components; therefore the total number of scalar variables is 16 .

Equations (1.1) to (1.8) consist of five vector equations and three scalar equations. Each vector equation consists of three scalar equations; hence the total number of scalar equations is 18 . Since the number of independent scalar equations should equal the number of scalar variables - two of the 18 equations should be dependent on the other equations.

We first prove that Gauss's magnetic flux law [its integral form is equation (A.5.4), $\oint_{A} \underline{B} \cdot \underline{d A}=0$ ] depends on Faraday's law [equation (A.1.1) $\left.\frac{d}{d t} \int_{A} \underline{B} \cdot \underline{d A}=-\oint_{s} \underline{E} \cdot \underline{d s}\right]$.

We select any volume $v o l$ surrounded by a closed surface $A$. Since the surface $A$ is closed - all of its boundaries $S$ converge to a point causing the right hand side line integral in equation (A.1.1) above to vanish. Therefore:

$$
\frac{d}{d t} \oint_{A} \underline{B} \cdot \underline{d A}=0
$$

It follows from equation (A.7.1) that if we divide the space into arbitrary volumes vol with outer envelope surfaces $A$ and these volumes move along according to the velocity vector field $V$ - the integral $\oint \underline{B} \cdot \underline{d A}$ over the outer envelope surfaces $A$ of each of these volumes

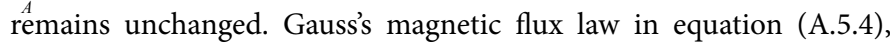
$\oint \underline{B} \cdot \underline{d A}=0$, serves as an initial condition to equation (A.7.1) stating that the initial value of these surface integrals cannot be selected arbitrarily. Their initial value must be 0 , and since their value remains unchanged they must equal 0 at all times. We thus obtain that at all times:

$$
\oint_{A} \underline{B} \cdot \underline{d A}=0
$$

Therefore, Gauss's magnetic flux law in equation (A.7.2) is not an independent equation. It is compatible with Faraday's law for closed surfaces [equation (A.7.1)] and serves as an initial condition to any closed surface integral $\oint_{A} \underline{B} \cdot \underline{d A}$. Since the time derivative of this integral vanishes [equation (A.7.1)] - its value equals 0 at all times. This dependence is valid in integral form [equation (A.5.4) on (A.1.1)] as well as in differential form [equation (1.3) on (1.1)].

We now prove that the charge conservation law in equation (1.5) is dependent on Ampere's law in equation (1.2) and Gauss's electricity flux law in equation (1.4). We do it by proving that equation (A.3.1), which is the integral form of the charge conservation law $\frac{d}{d t} \int_{V o l} \rho d(V o l)+\oint_{A} \underline{J} \cdot \underline{d A}=0$ , is dependent on equation (A.6.1), $\frac{d}{d t} \int_{A} \underline{D} \cdot \underline{d A}+\int_{A} \underline{J} \cdot \underline{d A}=\oint_{s} \underline{H} \cdot \underline{d s}$ which is the integral form of Ampere's law, and Gauss's electricity flux law in differential form $\nabla \cdot \underline{D}=\rho$, equation (1.4).

We refer again to Figure 7, in the particular case where the point $\mathrm{P}$ is far enough from the center $\mathbf{o}$ of the spherical surface so that the circular area converges to a point [see the two paragraphs preceding equation (A.6.4)]. In this particular case the integral form of Ampere's law is presented in equation (A.7.3):

$\frac{d}{d t} \oint_{A} \underline{D} \cdot \underline{d A}+\oint_{A} \underline{J} \cdot \underline{d A}=0$

But according to Gauss's theorem:

$\oint_{A} \underline{D} \cdot \underline{d A}=\int_{V o l}(\nabla \cdot \underline{D}) d(V o l)$

Substituting equation (A.7.4) into equation (A.7.3): 


$$
\frac{d}{d t} \int_{V o l}(\nabla \cdot \underline{D}) d(V o l)+\oint_{A} \underline{J} \cdot \underline{d A}=0
$$

According to Gauss's electricity flux law in differential form, equation (1.4):

$$
\nabla \cdot \underline{D}=\rho
$$

Substitution of equation (A.7.6) into equation (A.7.5) yields:

$$
\frac{d}{d t} \int_{V o l} \rho d(V o l)+\oint_{A} \underline{J} \cdot \underline{d A}=0
$$

Equation (A.7.7) is identical to the charge conservation law in equation (A.3.1), and is thus dependent on Ampere's law and Gauss's electricity flux law. This dependence is valid in differential form as well, namely: equation (1.5) is dependent on Ampere's law in equation (1.2) and Gauss's electricity flux law in equation (1.4).

We started out with 16 scalar variables and 18 scalar equations. Since two scalar equations are dependent on other equations - the number of independent equations equals 16. Therefore, the number of independent equations equals the number of variables and the necessary condition for the corrected Maxwell equations to be a welldefined mathematical problem is met. Consequently, for any particular problem a unique solution might exist which depends on the boundary and initial conditions.

It is important to note that had we applied equation (A.6.2) for Ampere's law - then, in the most general case where the velocity vector field varies space-wise, the charge conservation law (A.7.7) would not be dependent on the other equations. Consequently we would be stuck with 16 variables and 17 independent equations, where there would be no solution. This is another proof that equation (A.6.1), rather than equation (A.6.2), is the correct form of Ampere's law.

\section{The Faraday paradox}

The Faraday paradox is resolved by reference to the corrected Maxwell equations.

The significance of this section goes far beyond the resolution of the Faraday paradox. It is the basis for the resolution of many (most probably all) other paradoxes associated with Maxwell's equations. These paradoxes stem from the incompleteness of the common Maxwell's equations, i.e.: terms that they are missing. When the missing terms are restored to the corrected Maxwell equations - these paradoxes do not arise at the outset.

The Faraday paradox is presented at the following Internet link where the setup is shown in Figure 1.

Since the magnet is cylindrical, i.e.: axisymmetric, rotating the magnet does not change the magnetic field at any point in space, hence it does not have any effect on the measured voltage, which is compatible with the experiment results. The measured voltage can be influenced only by the rotation of the disc.

In the above link the Faraday paradox is resolved by applying the "Lorentz Force". So why is it still called a paradox? The problem is that the Faraday paradox cannot be resolved based only on Maxwell's equations (which should be the universal EM laws) but must rely on the Lorentz force which was introduced separately from Maxwell's equations.

However, as is shown in section A.4, the fact that the Lorentz force is not predicted by Maxwell's equations is due to the incompleteness of these equations. When applying the corrected Maxwell equations, where the missing terms are restored, the Lorentz force is a direct consequence of the corrected equations as shown by equation (A.4.17), and the Faraday paradox does not arise to begin with.

\section{Conclusion}

The soundness of this article can be checked through the answer to the following question: "Are Maxwell's equations incomplete?", namely: are some terms missing from these equations? If the answer is "no" this article is pointless. However, if the answer is "yes" - the theory of relativity collapses, as shown in the following logical flow-chart, since the article demonstrates beyond any doubt that Maxwell's equations are incomplete.

The theory of relativity was not readily accepted because it has contradicted common sense. But after more than a century of exposure to it the scientific community is absolutely certain about its unquestionable validity. However, we must go back to the good old Galilean transformation and Newtonian mechanics since this article clearly demonstrates that the theory of special relativity is based on incorrect notions, namely: forcing physical phenomena to comply with a wrong form of Maxwell's electromagnetic differential equations. Formulating the corrected Maxwell equations and solving them for planar EM waves in vacuum confirms (assuming that the integral laws which are the basis for Maxwell's equations are correct) that the Galilean transformation and Newtonian mechanics are valid, not only as low speed approximations but as exact laws. The corrected Maxwell equations might pave the way to the formulation of the long sought unified theory of mechanics and electromagnetism.

The theory of relativity is inconsistent (see flow-chart) which has led to many paradoxes and a large number of futile and costly experiments to "prove" its validity. [Nobody has found it necessary to prove Newton's laws or the laws of thermodynamics]. But the greatest damage of the theory of relativity may be related to the lack of progress in important engineering projects. The unavailability of commercial fusion energy, after many decades of intense efforts, is most probably due to the application of Maxwell's equations which are an inadequate form of the EM laws.

\section{Acknowledgments}

I would like to express my sincere gratitude to Nachum Bergos, Orrie Cohen, Yitzhak Kohn and Yaacov Mekler for their comments that made the article more easily readable. In particular I would like to thank Moshe Yarden, CEO of the Medisim Company, for dedicating many hours of his precious time to review and improve this article.

In addition, I would like to thank professor Moshe Einat of Ariel University and Harry Ricker and his physics Internet forum for their comments and criticism. Mr. Ricker's referral to publications by Branko Petrovic, whom I also thank very much enabled me to derive the complete electromagnetic equations corresponding to Faraday's and Ampere's laws in Appendix $A$.

\section{References}

1. Arnold S (1971) Electrodynamics, Lectures on Theoretical Physics, Academic Press, USA.

2. Smirnov VI (2014) A course of higher mathematics. Pregamon Press, USA.

Citation: Eisenman MN (2016) Back to Galilean Transformation and Newtonian Physics Refuting the Theory of Relativity. J Phys Math 7: 198. doi: 10.4172/20900902.1000198 